\title{
Transatlantica
}

Revue d'études américaines. American Studies Journal

$2 \mid 2011$

Sport et société / Animals and the American

Imagination

\section{Conférence de Nina Crimm}

Université Paris-Est Créteil - juin 2011

\section{Elizabeth LEVY}

\section{(2) OpenEdition}

\section{Journals}

Édition électronique

URL : https://journals.openedition.org/transatlantica/5688

DOI : 10.4000/transatlantica. 5688

ISSN : 1765-2766

Éditeur

Association française d'Etudes Américaines (AFEA)

\section{Référence électronique}

Elizabeth LEVY, "Conférence de Nina Crimm », Transatlantica [En ligne], 2 | 2011, mis en ligne le 17 juin 2012, consulté le 09 février 2023. URL : http://journals.openedition.org/transatlantica/5688; DOI https://doi.org/10.4000/transatlantica.5688

\section{Ce document a été généré automatiquement le 9 février 2023}

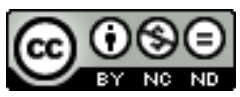

Creative Commons - Attribution - Pas d'Utilisation Commerciale - Pas de Modification 4.0 International - CC BY-NC-ND 4.0

https://creativecommons.org/licenses/by-nc-nd/4.0/ 


\title{
Conférence de Nina Crimm
}

\author{
Université Paris-Est Créteil - juin 2011
}

\author{
Elizabeth LEVY
}

1 Au mois de juin 2011, l'équipe du laboratoire IMAGER (Institut des Mondes Anglophone, Germanique et Roman) de l'Université Paris-Est Créteil a reçu Nina Crimm comme professeur invité. Professeur de droit à St. John's University à New York, le professeur Crimm est spécialiste des questions fiscales aux États-Unis et de la manière dont cellesci s'articulent avec la Constitution américaine. Au cours de son séjour à Paris, elle a donné à l'Université de Créteil une communication intitulée «When Religion, Parochial Schools and Taxes Meet the First Amendment ", qui visait à mettre en lumière les rapports complexes qu'entretiennent les écoles religieuses et les questions fiscales dans le cadre $\mathrm{du}$ respect du premier amendement de la Constitution aux États-Unis.

Dès le dix-huitième siècle les pères fondateurs avaient envisagé les églises et les écoles comme des lieux de création de l'identité américaine par le biais de la diffusion des valeurs, leur conférant ainsi un rôle de garants de la stabilité des institutions et de la démocratie. Toutefois, si l'État permettait une meilleure diffusion des savoirs en aidant financièrement les écoles laïques, les lieux de cultes, eux, devaient rester des institutions privées et ne pouvaient recevoir d'aide financière de l'État. L'apparition et le développement, notamment au dix-neuvième siècle, d'un grand nombre d'écoles catholiques suscitèrent de nouvelles questions. Ces écoles, puisqu'elles suivaient les mêmes programmes que les écoles laïques tout en ayant un fondement religieux, présentaient un cas particulièrement ambigu et problématique : quelle politique fiscale fallait-il adopter vis-à-vis d'elles ?

D'après la Constitution de 1788 , le Congrès a les pleins pouvoirs en matière d'impôt, et il lui revient de déterminer comment celui-ci est prélevé aussi bien pour les entités laïques que pour les entités religieuses. Le premier amendement de la Constitution stipule aussi que «Le Congrès ne fera aucune loi qui touche l'établissement ou interdise le libre exercice d'une religion. ", décourageant ainsi tout lien ou rapprochement excessif entre l'Église et l'État. Dès lors, la question des écoles religieuses aux États-Unis se pose encore de nos jours sous deux angles différents: il s'agit de savoir, d'une part, si le 
Congrès doit contraindre les écoles religieuses à payer des impôts, et, d'autre part, si le gouvernement pouvait pratiquer une aide financière dite « indirecte ».

Malgré les pleins pouvoirs qui lui étaient octroyés en la matière, le Congrès s'est toujours montré parcimonieux au sujet des impôts depuis la guerre de Sécession. Jugeant que les lieux de cultes, les écoles et les organisations de charité n'avaient plus de revenu imposable une fois qu'ils avaient rempli les missions d'aide, d'éducation et/ ou de charité qui étaient les leurs, le Congrès choisit d'exempter ces institutions par principe. La section 501c3 du Internal Revenue Code entérine cette décision, qui est conforme au premier amendement, mais qui n'est cependant pas rendue obligatoire par ce dernier.

5 La question de l'exemption des écoles religieuses est cependant complexe. Si en effet les lieux de cultes sont automatiquement exemptés d'impôts, il en va autrement pour les écoles, qui doivent prouver à l'IRS (International Revenue Service) qu'elles ne procèdent pas à la discrimination raciale lorsqu'elles sélectionnent leurs étudiants. Or, par définition, un grand nombre d'écoles religieuses sont tournées vers une seule religion et ont un comportement qui peut être considéré comme sectaire. De plus, en règle générale, les écoles religieuses ont le droit de recevoir des «cadeaux » ou dons, qui rendent l'idée selon laquelle elles n'ont pas de revenus imposables caduque. De fait, une partie de l'opinion s'interroge sur cette exemption et se demande si celle-ci ne s'apparente pas à une forme de favoritisme dont bénéficieraient les écoles religieuses aux dépens du contribuable américain.

De la même manière, les débats au sujet de l'aide financière indirecte apportée par l'État aux écoles religieuses ont été nombreux. Une aide financière directe de l'État ne saurait que très difficilement être conforme au premier amendement, puisqu'elle reviendrait implicitement pour l'État à aider une religion au détriment d'autres. D'aucuns considèrent que le premier amendement interdit que l'argent du contribuable américain ne soit utilisé pour financer une quelconque activité religieuse sans son accord. La question principale concerne par conséquent l'aide financière indirecte que l'État peut apporter aux écoles religieuses. Cette aide financière prend le plus communément la forme de bons (vouchers) distribués aux parents d'élèves, qui peuvent les utiliser pour financer la scolarité de leurs enfants dans l'école de leur choix, que celle-ci soit laïque ou religieuse.

7 La Cour suprême a récemment défendu plusieurs programmes de distribution de bons grâce auxquels un grand nombre d'écoles religieuses ont pu avoir accès à des fonds gouvernementaux. En 2002, l'arrêt Zelmam v. Simmons établit la constitutionnalité d'un programme d'aide financière indirecte aux écoles développé dans l'ohio et dont $82 \%$ des bénéficiaires étaient des écoles religieuses. Selon le juge Rehnquist, le programme avait des visées laïques et neutres puisqu'il était destiné à garantir une meilleure éducation aux habitants de l'état. Tel qu'il était conçu, le programme n'était pas destiné à favoriser une religion par rapport à une autre ou une école religieuse par rapport à une école laïque. Le programme ne pouvait être jugé anticonstitutionnel même si les écoles qui recevaient l'aide gouvernementale étaient majoritairement religieuses, dans la mesure où les personnes responsables de cet état de fait étaient les parents d'élèves. En décidant du type d'écoles dans lesquelles elles souhaitaient placer leurs enfants, les familles ne faisaient qu'exercer leur liberté et leur libre arbitre, valeurs fondamentales qui ne sauraient être restreintes dans ce cas de figure. La décision la plus récente de la 
Cour suprême au sujet des écoles religieuses, qui remonte à avril 2011, va dans le même sens.

8 Nina Crimm a terminé sa conférence en rappelant à quel point l'éducation et les questions fiscales avaient été imbriquées au cours de l'histoire américaine. Ayant une forte dimension technique et administrative, les conflits que ces liens peuvent susciter sont néanmoins cruciaux car ils touchent en réalité au cœur même de l'identité américaine. Les enjeux dépassent le cadre de la simple utilisation de l'argent et éveillent au contraire des remises en questions des valeurs américaines, des croyances individuelles et des droits définis par la Constitution, dont l'interprétation peut différer.

9 C'est précisément sur la question des interprétations de la Constitution que le débat s'engagea après la présentation de Nina Crimm. Paul Secunda, spécialiste du droit américain du travail et des lois sur l'éducation, professeur de droit invité à l'Université de Nanterre au cours du mois de juin 2011 et professeur à Marquette University School of Law dans le Wisconsin, revint sur l'idée du libre arbitre des familles telle qu'elle avait été évoquée par le professeur Crimm. Il rappela qu'il y a à l'heure actuelle au moins deux manières concurrentes de comprendre le premier amendement de la Constitution américaine. Pour certains, ce texte fondateur instaure la «neutralité » de l'État, interdisant à ce dernier de privilégier une religion au détriment d'une ou des autres, mais ne lui interdisant pas pour autant d'entrer en contact avec ces dernières. Pour d'autres, le premier amendement signifie qu'il existe un «mur de séparation » entre l'Église et l'État.

10 Paul Secunda affirma clairement qu'il faisait partie des gens qui considèrent qu'il doit exister, comme l'avait formulé Jefferson, un «mur de séparation » entre le religieux et le politique aux États-Unis et revint sur l'arrêt Zelman et ses conséquences pour introduire le concept de "néo-formalisme ". Il rappela que la Cour suprême américaine avait, jusqu'aux années 1980, privilégié une lecture de la Constitution qui favorisait une séparation stricte entre l'Église et l'État mais qu'un revirement en faveur de la neutralité s'était opéré ces trente dernières années. D'après lui, l'accent mis sur le choix des familles par les juges conservateurs de la Cour suprême visait à mettre en place une stratégie oblique: mettre en avant un souci formel de défense des libertés individuelles permettait en fait de rendre acceptables constitutionnellement des liens trop étroits entre l'Église et l'État. Ainsi, dans le cas des écoles religieuses, en insistant sur le libre arbitre et la possibilité qui devait être donnée aux citoyens américains de faire l'usage qu'ils souhaitaient de leur argent, la Cour en venait à légitimer une pratique anticonstitutionnelle. Dans les faits, que l'aide soit indirecte ou pas, le résultat était le même : des écoles religieuses recevaient des fonds gouvernementaux avec l'aval de la Cour suprême, ce qui était pourtant précisément ce que le premier amendement tel que Paul Secunda l'interprétait visait à empêcher.

11 Nina Crimm revint sur la question de l'existence du mur de séparation entre l'Église et l'État et affirma qu'elle était d'accord avec l'idée que des brèches avaient été ouvertes au sein de celui-ci. Quant à savoir si ces marques d'effondrement étaient inquiétantes, elle rappela que la Constitution visait à garantir le pluralisme religieux et que c'était principalement avec la diversité religieuse que l'État n'avait pas le droit d'intervenir. Cependant, elle invita aussi à la méfiance face à des concepts tels que celui de «néoformalisme ». Pour elle, de tels termes n'étaient en réalité que des étiquettes réductrices destinées à prendre les gens par les sentiments et à les inquiéter. Elle ajouta 
que la question de ce qui est autorisé ou non dans le cadre du premier amendement est extrêmement complexe et nécessite une réflexion à plusieurs niveaux, que des termes tels que « néo-formalisme » ne permettent pas.

La discussion s'engagea ensuite sur les différences et similitudes qui pouvaient exister en France et aux États-Unis au sujet des questions religieuses. Les débats rappelèrent combien les situations en France et aux États-Unis pouvaient sembler être les deux revers d'une même médaille. Les deux pays s'interrogent sur la meilleure manière de faire cohabiter des religions différentes sur le même sol ; mais alors qu'en France les interrogations principales tournent en ce moment autour des minorités religieuses et la place croissante qu'elles occupent aux yeux de certains, les interrogations aux ÉtatsUnis concernent la trop grande place que la religion majoritaire peut prendre.

13 L'intérêt suscité par les comparaisons entre la France et les États-Unis au sein de l'assistance qui était composée de Français et d'Américains s'explique probablement par le fait que, comme il le fut rappelé pour clôturer la séance, les questions et querelles religieuses sont à la fois très abstraites et très concrètes et qu'elles ont, pour cette raison, de profondes ramifications dans l'identité et la nature des sociétés qu'elles concernent. En effet, si les débats se révélèrent passionnants en raison du grand nombre d'exemples concrets qui furent évoqués et au sujet desquels chacune des personnes présentes semblait avoir quelque chose à ajouter (comme le traitement différent réservé à l'Église de scientologie d'un pays à l'autre ou la nécessité pour certains d'une laïcité de combat...), ils le furent aussi car ils montrèrent à quel point il était en revanche difficile à chacun d'établir un cadre théorique cohérent et dont l'objectivité ne puisse pas être remise en cause. Le concept de "néo-formalisme" développé par Paul Secunda ainsi que les réponses qu'il suscita de la part du professeur Crimm étaient en eux-mêmes très révélateurs du fait que tout raisonnement en matière de relation entre l'Église et l'État tendait à devenir quasiment immanquablement une posture militante qui pouvait être revendiquée ou remise en question en tant que telle.

Si l'on va plus loin, l'angle de la fiscalité est particulièrement intéressant dans la mesure où il replace justement le débat dans un cadre concret, tout en montrant à quel point celui-ci est rapidement dépassé. Si la question de l'impôt et des écoles religieuses aux États-Unis peut paraître secondaire à un public de néophytes, force est de constater qu'elle a en réalité de nombreuses répercussions pratiques et théoriques. En effet, les écoles religieuses américaines et leur traitement constituent en général un laboratoire d'expérimentations juridiques dont la portée est loin d'être restreinte. Ainsi, les « faithbased initiatives" qui constituaient la clef de voûte de la politique d'aide sociale de George W. Bush, en entérinant l'idée que la meilleure manière de lutter contre la pauvreté aux États-Unis consistait pour l'État à verser des subventions à des groupes religieux qui avaient développé des programmes d'aide sociale performants, ont en très grande partie été rendues possibles et étendues par les récents arrêts de la Cour suprême au sujet des bons évoqués plus haut. Étant donné que l'argent de l'État pouvait être utilisé indirectement pour financer la scolarité d'élèves d'écoles religieuses, le même type de raisonnement pouvait être appliqué aux organisations de charité. De cet enchaînement de situations concrètes découle non seulement des changements tangibles des politiques éducatives ou sociales du pays, mais aussi un changement du cadre théorique. Alors que Paul Secunda rappelait la coexistence de la théorie de la séparation et de la théorie de la neutralité eu égard au premier amendement de la Constitution américaine, il faut aussi noter qu'un glissement semble s'être amorcé et 
qu'un nouveau courant de lecture, mettant l'accent sur l'idée de collaboration, a commencé à émerger.

15 La question de la fiscalité et des écoles religieuses était donc non seulement l'occasion de débats animés, mais aussi l'occasion d'un rappel implicite de l'extrême souplesse du texte constitutionnel américain qui, quoi qu'intangible clef de voûte de la société américaine depuis la fin du XvIII ${ }^{e}$ siècle, se caractérise aussi par la grande complexité et concurrence des interprétations qu'il peut susciter et des différents projets d'organisations sociales, institutionnelles et juridiques qu'il peut sous-tendre.

INDEX

Thèmes : Actualité de la recherche

\section{AUTEUR}

\section{ELIZABETH LEVY}

Université Paris Diderot-Paris VII 